\title{
EOPS: clopidogrel versus aspirina mas inhibidores de la bomba de protones en paciente coronario con antecedente de úlcera gástrica sangrante inducida por aspirina.
}

Hornstein L. Eops. clopidogrel versus aspirina mas inhibidores de la bomba de protones en paciente coronario con antecedente de úlcera gástrica sangrante inducida por aspirina.. Evid. actual. práct. ambul. 2005;8:183-184.

\section{Escenario clínico}

Un paciente de 66 años con angina crónica estable en tratamiento crónico con aspirina $325 \mathrm{mg}$ por día presentó una hemorragia digestiva con shock hipovolémico que requirió asistencia respiratoria mecánica. Se diagnosticó una úlcera gástrica sangrante inducida por aspirina. Dada la indicación absoluta de tratamiento antiagregante plaquetario por su riesgo coronario, una vez curado de su úlcera gástrica, el médico que lo atiende se plantea cuál es la mejor estrategia a seguir.

\section{Pregunta que generó el caso:}

¿En los pacientes con antecedentes de úlcera gástrica sangrante con indicación absoluta de terapia antiagregante (población), el uso de clopidogrel es más seguro que el uso de aspirina sumado a un inhibidor de la bomba de protones (intervención y comparación), para la prevención de la recurrencia de la úlcera sangrante (resultado)?

\section{Estrategia de búsqueda:}

Se realizó una búsqueda en Medline limitando la búsqueda a ensayos clínicos aleatorizados, revisiones sistemáticas y metaanálisis. Se emplearon como palabras clave: "clopidogrel aspirin and ulcer bleeding".Se encontró un ensayo clínico aleatorizado que parecía apropiado para responder la pregunta.

\section{Terapias antiplaquetarias y riesgo de sangrado gastrointestinal}

La aspirina dobla el riesgo de sangrado gastrointestinal aún en dosis de $75 \mathrm{mg}$ día ${ }^{1-2}$. La historia de hemorragia digestiva alta por una úlcera es el factor de riesgo más importante para recidiva de sangrado en pacientes que toman aspirina. ${ }^{3,4}$. Hasta un $15 \%$ de los pacientes tomadores de aspirina con historia de sangrado gastrointestinal tienen sangrado recurrente dentro de los 5 años.

El uso concomitante de inhibidores de la bomba de protones reduce el riesgo, pero la adherencia a este régimen puede limitar su uso en pacientes que en general toman varias medicaciones. ${ }^{6.9}$. Una alternativa es reemplazar la aspirina por otro antiagregante plaquetario como el clopidogrel. El clopidogrel es un antitrombótico efectivo dado que bloquea la activación plaquetaria por la adhesión irreversible al receptor del difosfato de adenosina de las plaquetas. Esto a su vez previene la activación del complejo glicoprotéico IIb/IIla, principal receptor plaquetario para el fibrinógeno.

En el estudio CAPRIE ensayo clínico aleatorizado dónde se comparó la efectividad de $325 \mathrm{mg}$ de aspirina versus $75 \mathrm{mg}$ de clopidogrel para la prevención secundaria de eventos vasculares trombóticos, el clopidogrel fue marginalmente mas efectivo que la aspirina y tuvo una tasa moderadamente menor de sangrado gastrointestinal $(5 \% \text { vs } 7 \%)^{10}$. Las guías cardiológicas actuales recomiendan el uso de clopidogrel en pacientes hospitalizados por síndrome coronario agudo con intolerancia gastrointestinal mayor a la aspirina ${ }^{11}$, sin embargo el uso de clopidogrel como alternativa al uso de aspirina mas inhibidores de la bomba de protones en pacientes con riesgo de úlcera no había sido estudiado prospectivamente hasta este ensayo.

\section{Resumen de la evidencia}

Chan FKL, Ching JYL, Hung LCT, et al. Clopidogrel versus aspirin and esomeprazole to prevent recurrent ulcer bleeding. N Engl $J$ Med 2005;352:238-244.
Objetivo del estudio: comparar clopidogrel con aspirina más esomeprazol para la prevención de sangrado gastrointestinal recurrente en pacientes de alto riesgo.

Diseño: ensayo clínico aleatorizado doble ciego de 12 meses de duración

\section{Ambito: Prince of Wales Hospital de Hong Kong}

Población: 320 pacientes adultos, de ambos sexos, con antecedentes de consumo de $325 \mathrm{mg}$ de aspirina para la prevención de enfermedad vascular, con historia de sangrado por úlcera gastrointestinal inducida por aspirina, una vez curada la misma (por endoscopia) y con test negativo para H. pylori. Media de edad 72 años.

Intervención: Luego de la curación endoscópica de la úlcera y de la erradicación del $\mathrm{H}$. pylori, los pacientes fueron asignados aleatoriamente a recibir $75 \mathrm{mg}$ diarios de clopidogrel mas esomeprazol placebo (161 pacientes) o 80mg diarios de aspirina mas esomeprazol $20 \mathrm{mg}$ dos veces al día (159 pacientes) por 12 meses. Los pacientes fueron reevaluados al mes, a los 3 meses y luego cada 3 meses hasta el final del estudio.

En cada visita se midió el nivel de hemoglobina $(\mathrm{Hb})$, la adherencia al tratamiento (conteo de pastillas), utilización de otras drogas y la seguridad (examen clínico, análisis de laboratorio y reporte de efectos adversos). Los pacientes que abandonaron el tratamiento fueron seguidos hasta el final del estudio.

Medición de resultados principales: El punto final fue recurrencia del sangrado por úlcera gastrointestinal: hematemesis o melena documentada por el médico con úlcera o erosiones confirmadas por endoscopia, o descenso en el nivel de $\mathrm{Hb}$ de por lo menos de $2 \mathrm{~g} / \mathrm{dl}$ en presencia de úlcera o erosiones endoscópicas.

El punto final secundario fue el sangrado gastrointestinal bajo, definido como melena o sangrado rectal con requerimiento de internación o transfusión, con endoscopia alta negativa, o descenso del nivel de $\mathrm{Hb}$ por lo menos de $2 \mathrm{~g} / \mathrm{dl}$ en presencia de SOMF positiva y endoscopia alta negativa.

Resultados: el sangrado recurrente por úlcera gastrointestinal ocurrió en 13 pacientes que recibían clopidogrel y en 1 de los que recibía aspirina mas esomeprazol.

La incidencia acumulativa de sangrado recurrente durante los 12 meses de seguimiento fue de $8.6 \%$ (IC95\% de 4.1 a $13.1 \%$ ) para los pacientes que recibieron clopidogrel y de $0.7 \%$ (IC95\% de 0 a $2.0 \%$ ) para los que recibieron aspirina mas esomeprazol. La diferencia fue de $7.9 \%$ con un IC 95\% para la diferencia de 3.4 a $12.4 \%$; $p=0.001$.

La incidencia acumulativa de sangrado gastrointestinal bajo fue de $4.6 \%$ (IC $95 \%$ de 1.3 a $7.9 \%$ ) para el grupo clopidogrel y de $4.6 \%$ (IC $95 \%$ de 1.3 a $8 \%$ ) en el grupo aspirina mas esomeprazol $(p=0.98)$. La incidencia de eventos isquemicos no difirió significativamente entre los dos grupos. La mortalidad fue de 8 pacientes en el grupo clopidogrel y de 4 pacientes en el grupo aspirina más esomeprazol.

El análisis fue hecho por intención de tratar. La tasa de seguimiento fue similar en ambos grupos y las causas de abandono del tratamiento también.

Conclusión de los autores: entre los pacientes con historia de sangrado gastrointestinal inducido por aspirina en los que la úlcera ha curado antes de recibir el tratamiento en estudio, aspirina mas esomeprazol fue superior al clopidogrel para prevenir la recurrencia de sangrado por úlcera gastrointestinal.

Los resultados de este trabajo no apoyan la recomendación habitual de que los pacientes con intolerancia mayor gastrointestinal a la aspirina deben recibir clopidogrel. 


\section{Comentario y conclusiones para la práctica}

La evidencia actual sobre la seguridad gastrointestinal del clopidogrel deriva de análisis secundarios de estudios que no fueron diseñados específicamente para medir este resultado y en los cuales la dosis de aspirina utilizada era mayor $(325 \mathrm{mg})$. Este estudio plantea claramente la duda sobre la seguridad del clopidogrel en pacientes con antecedentes de sangrado gastrointestinal alto inducido por aspirina. La dosis óptima de inhibidores de la bomba de protones para pacientes con alto riesgo en el uso de aspirina no está clara. El estudio no discute si existen variaciones genéticas entre grupos étnicos o raciales que puedan tener algún efecto en el riesgo de sangrado o la eficacia del tratamiento. Por último, el hecho de que las drogas fueran re-empaquetadas puede haber modificado de alguna forma la absorción y con esta la eficacia terapéutica o los efectos adversos de las mismas. En síntesis, no parece adecuado reemplazar el uso de aspirina más inhibidores de la bomba de protones por clopidogrel en pacientes con alto riesgo de sangrado gastrointestinal. Dado la importante diferencia de sangrado observada en el grupo AAS + IBP es válido preguntarse cuan comparables son las intervenciones ya que existe un indiscutible beneficio atribuible al esomeprazol. Si bien sería interesante realizar un estudio que compare ambas drogas más inhibidores de la bomba de protones en este tipo de pacientes, el estudio confirma que el uso de aspirina + un inhibidor de la bomba de protones es muy seguro y el potencial beneficio adicional del clopidogrel, si existiera, no justificaría los costos de su utilización.

Lucila Hornstein [ Becaria de Gerenciamiento. Plan de Salud. Hospital Italiano de Buenos Aires. ]

\section{Referencias:}

1. Derry S, Loke YK. Risk of gastrointestinal haemorrhage with long term use of aspirin: meta-analysis. BMJ 2000:321:1183-1187.

2. Weil J, Colin-Jones D, Langman M, et al. Prophylactic aspirin and risk of peptic ulcer bleeding. BMJ 1995;310:827-830.

3. Garcia Rodriguez LA, Jick H. Risk of upper gastrointestinal bleeding and perforation associated with individual non-steroidal anti-inflammatory drugs. Lancet 1994;343:769-772. [Erratum, Lancet 1994;343:1048.][ISI][Medline]

4. Lanas A, Bajador E, Serrano P, et al. Nitrovasodilators, low-dose aspirin, other nonsteroidal antiinflammatory drugs, and the risk of upper gastrointestinal bleeding. $\mathrm{N}$ Engl $\mathrm{J}$ Med 2000;343:834-839.

5. Lai KC, Lam SK, Chu KM, et al. Lansoprazole for the prevention of recurrences of ulcer complications from long-term low-dose aspirin use. N Engl J Med 2002;346:2033-2038. 6. Garcia Rodriguez LA, Ruigomez A. Secondary prevention of upper gastrointestinal bleeding associated with maintenance acid-suppressing treatment in patients with peptic ulcer bleed. Epidemiology 1999;10:228-232.

7. Chan FK, Chung SC, Suen BY, et al. Preventing recurrent upper gastrointestinal bleeding in patients with Helicobacter pylori infection who are taking low-dose aspirin or naproxen. N Engl J Med 2001:344:967-973.

8. Laine L. Approaches to nonsteroidal anti-inflammatory drug use in the high-risk patient. Gastroenterology 2001:120:594-606.

9. Peura DA, Malfertheiner P. Dichotomies and directions in acid-related disorders. Aliment Pharmacol Ther 2004;19:Suppl 1:77-80.

10.CAPRIE Steering Committee. A randomised, blinded, trial of clopidogrel versus aspirin in patients at risk of ischaemic events (CAPRIE) Lancet 1996-348:1329-1339.

11. Braunwald E, Antman EM, Beasley JW, et al. ACC/AHA 2002 guideline update for the management of patients with unstable angina and non-ST-segment elevation myocardial infarction -- summary article: a report of the American College of Cardiology/American Heart Association task force on practice guidelines (Committee on the Management of Patients with Unstable Angina). J Am Coll Cardiol 2002;40:1366-1374.

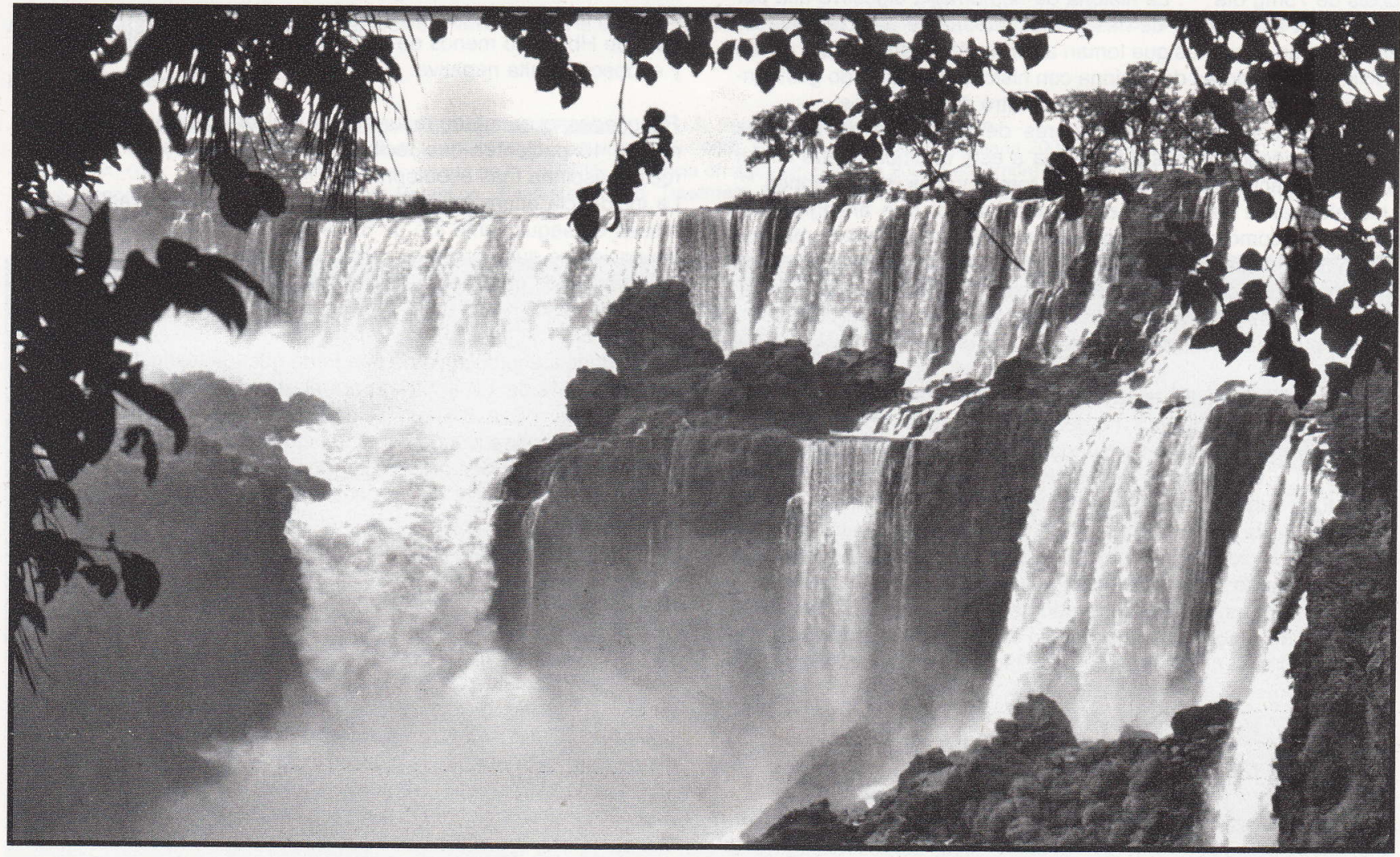

Gentileza Secretaria de Turismo de La Nación 